\title{
MULTI-MODAL SENSOR LOCALIZATION USING A MOBILE ACCESS POINT
}

\author{
Brian M. Sadler ${ }^{1}$, Richard J. Kozick ${ }^{2}$, Lang Tong ${ }^{3}$ \\ ${ }^{1}$ Army Research Laboratory, Adelphi, MD 20783, bsadler@arl.army.mil \\ ${ }^{2}$ Bucknell University, Lewisburg, PA 17837, kozick@bucknell.edu \\ ${ }^{3}$ Cornell University, EECS Department, Ithaca, NY
}

\begin{abstract}
We consider the problem of sensor node localization in a randomly deployed sensor network, using a mobile access point (AP). The mobile AP can be used to localize many sensors simultaneously in a broadcast mode, without a preestablished sensor network. We consider a multi-modal approach, combining radio and acoustics. The radio broadcasts timing, location information, and acoustic signal parameters. The acoustic emission may be used at the sensor to measure Doppler stretch, time delay, and angle of arrival. These measurements are individually sufficient to localize a sensor node, or they may be advantageously combined. We focus on the cases of Doppler and time delay. Sensor localization algorithms are developed, and performance analysis includes acoustic propagation effects caused by the turbulent atmosphere.
\end{abstract}

\section{INTRODUCTION}

Many sensor network scenarios call for random deployment, but require the sensor nodes to have known location and orientation, so that post-deployment techniques are needed for self-localization. This can be achieved by using beacons, which may be external to the network (such as GPS), or deployed within the network. In this paper, we consider the problem of sensor node localization in a randomly deployed sensor network, using a mobile access point (AP).

Deploying fixed beacons within the network enables a solution based on message passing between the nodes, e.g., see Moses et al. [1]. These beacons may be radio, or other modality such as acoustic, to take advantage of the sensor capability. This approach assumes the communications network is pre-established, and requires sufficient density of beacons to be deployed, which raises the complexity of at least some of the nodes. With random deployment, some nodes may be disadvantaged due to local sparsity of the beacons and/or node neighbors, and this can only be corrected if more nodes or beacons are deployed.

As an alternative, we consider the use of a mobile AP for both communications and beaconing. The mobile AP can be used to localize many sensors simultaneously in a broadcast mode, without communications or synchronization between the nodes. The localization algorithms require only that the nodes receive the AP broadcast, and the AP can provide many beaconing positions. Nodes can be localized during deployment (perhaps from the same platform as the mobile AP), or when more nodes are added to an existing network. Uplink communications may be desirable, e.g., to request more beaconing to reduce the localization error to a desired tolerance.

At least three different measurements at the sensor node can be used for node localization: Doppler shift, range via time delay estimation (TDE), and angle of arrival (AOA). These measurements, combined with AP mobility, are individually sufficient to estimate the node location; combinations of these may also be used. The AP, via broadcast, sends timing information, its location information, and emits an appropriate signal for measurement at the sensor node. This assumes the AP has an accurate determination of its own location and motion.

The above procedure can in principle be carried out with a single transmission modality, such as radio. However, the use of radio alone has some drawbacks. Radio frequency Doppler shift is not large for the typical, relatively low, AP velocity. Accurate TDE measurements require large timebandwidth product waveforms, which in turn implies a relatively sophisticated radio, whereas many sensor networks may rely on relatively low bandwidth communications to reduce both complexity and energy consumption in the sensor node. And, AOA measurement will rely on an antenna array, which again significantly raises the radio complexity.

Here we consider multi-modal transmission, radio and acoustic, with the acoustic waveform used for measuring Doppler, TDE, and/or AOA. Acoustic propagation velocity is slow, and large fractional bandwidths can be utilized. On the other hand, acoustic emission can be strongly affected by atmospheric turbulence, and higher frequencies attenuate rapidly. We assume the AP emits an appropriate acoustic signal, which may be synthetically generated, or result from the inherent platform noise (such as engine noise from a helicopter). In both cases, the emitted acoustic waveform may also be transmitted to the sensor node via radio, thus 
providing a reference for signal processing.

In this paper, we focus on Doppler and TDE, which requires only a single acoustic sensor at each node; other cases and combinations will be reported elsewhere. Acoustic experiments with AOA and a mobile access point are reported in [2].

\section{DOPPLER AND SENSOR LOCALIZATION}

We consider acoustic tone(s) emission from the mobile AP, derive algorithms for sensor node location estimation, and provide localization accuracy analysis that includes acoustic frequency, range, and propagation conditions. The source and sensor geometry is shown in Figure 1, with the unknown sensor location in 2-D denoted $\mathbf{x}_{o}=\left(x_{o}, y_{o}\right)$. Let the emitted acoustic frequency be $f_{s}$, the source location at time $t_{n}$ be $\mathbf{x}_{n}=\left(x_{n}, y_{n}\right)$, and the source velocity at time $t_{n}$ be $\dot{\mathbf{x}}_{n}=\left(\dot{x}_{n}, \dot{y}_{n}\right)$. The range vector from source to sensor is $\mathbf{r}_{n}=\mathbf{x}_{o}-\mathbf{x}_{n}$, the range is $r_{n}=\left\|\mathbf{r}_{n}\right\|$, and the source speed is $V_{n}=\left\|\dot{\mathbf{x}}_{n}\right\|$, where $\|\cdot\|$ denotes the Euclidean norm of the vector. In Figure 1, the angles $\alpha_{n}$ (between the source velocity vector and the range vector) and $\phi_{n}$ (the azimuth) are

$$
\begin{aligned}
\cos \alpha_{n} & =\frac{\mathbf{r}_{n} \cdot \dot{\mathbf{x}}_{n}}{r_{n} V_{n}} \\
\cos \phi_{n} & =\frac{x_{n}-x_{o}}{r_{n}}, \quad \sin \phi_{n}=\frac{y_{n}-y_{o}}{r_{n}},
\end{aligned}
$$

where $\cdot$ denotes the inner product between vectors. The Doppler-shifted frequency observed at the sensor node is given by [3]

$$
\begin{aligned}
f_{n} & =f_{s}\left(1+\frac{V_{n} \cos \alpha_{n}}{c}\right) \\
& =f_{s}+\left(\frac{f_{s}}{c}\right) \frac{\left(x_{o}-x_{n}\right) \dot{x}_{n}+\left(y_{o}-y_{n}\right) \dot{y}_{n}}{\left[\left(x_{o}-x_{n}\right)^{2}+\left(y_{o}-y_{n}\right)^{2}\right]^{1 / 2}} \\
& =f_{s}+\left(\frac{f_{s}}{c}\right) g\left(\mathbf{x}_{o} ; \mathbf{x}_{n}, \dot{\mathbf{x}}_{n}\right) .
\end{aligned}
$$

In (3)-(5), the unknowns are the sensor node location $\mathbf{x}_{o}=$ $\left(x_{o}, y_{o}\right)$, while the quantities $f_{s}, x_{n}, y_{n}, \dot{x}_{n}, \dot{y}_{n}$ are known because they are transmitted via radio from the AP to the sensor node. We assume the acoustic velocity $c$ is known. This can be obtained from measurements of meteorological parameters such as temperature, humidity, and so on; e.g., see [4]. The function $g\left(\mathbf{x}_{o} ; \mathbf{x}_{n}, \dot{\mathbf{x}}_{n}\right)$ is defined in (5) to emphasize that the sensor location $\mathbf{x}_{o}$ is unknown while the source parameters $\mathbf{x}_{n}, \dot{\mathbf{x}}_{n}$ are known.

In order to estimate the sensor location based on measurements of the Doppler-shifted frequency, it is clear from (3) and (4) that at least two measurements are required, with different source locations and/or trajectories. We consider

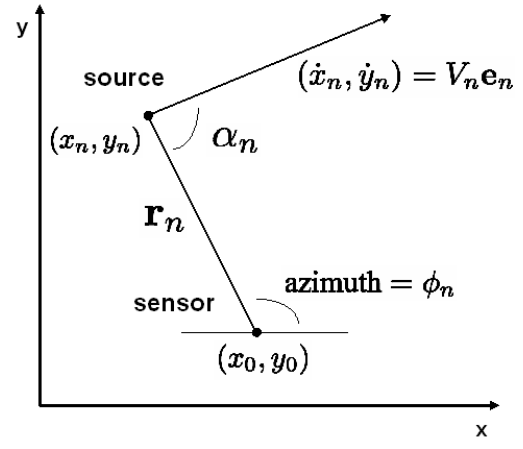

Fig. 1. Geometry of sensor and source.

the following model for $N$ measurements of the Doppler shift, $\Delta f_{n}=f_{n}-f_{s}$ :

$$
\Delta f_{n}=\left(\frac{f_{s}}{c}\right) g\left(\mathbf{x}_{o} ; \mathbf{x}_{n}, \dot{\mathbf{x}}_{n}\right)+\epsilon_{n}, n=1, \ldots, N .
$$

We model the estimation errors, $\epsilon_{1}, \ldots, \epsilon_{N}$, as independent, zero-mean, Gaussian random variables, with $\epsilon_{n} \sim$ $N\left(0, \sigma_{n}^{2}\right)$. The maximum likelihood estimate of sensor node location is given by weighted, nonlinear least-squares,

$$
\widehat{\mathbf{x}}_{o}=\underset{\mathbf{x}_{o}}{\arg \min } \sum_{n=1}^{N} \frac{1}{\sigma_{n}^{2}}\left[\Delta f_{n}-\frac{f_{s}}{c} g\left(\mathbf{x}_{o} ; \mathbf{x}_{n}, \dot{\mathbf{x}}_{n}\right)\right]^{2}
$$

The Fisher information matrix (FIM) for the sensor location parameter vector $\mathbf{x}_{o}$ is $\mathbf{J}=\left[\begin{array}{ll}J_{x x} & J_{x y} \\ J_{x y} & J_{y y}\end{array}\right]$, where

$$
\begin{aligned}
J_{x x}= & \sum_{n=1}^{N}\left(\frac{f_{s}}{c \sigma_{n}}\right)^{2}\left(\frac{\dot{x}_{n}+V_{n} \cos \alpha_{n} \cos \phi_{n}}{r_{n}}\right)^{2} \\
J_{y y}= & \sum_{n=1}^{N}\left(\frac{f_{s}}{c \sigma_{n}}\right)^{2}\left(\frac{\dot{y}_{n}+V_{n} \cos \alpha_{n} \sin \phi_{n}}{r_{n}}\right)^{2} \\
J_{x y}= & \sum_{n=1}^{N}\left(\frac{f_{s}}{c \sigma_{n}}\right)^{2}\left(\frac{\dot{x}_{n}+V_{n} \cos \alpha_{n} \cos \phi_{n}}{r_{n}}\right) \\
& \times\left(\frac{\dot{y}_{n}+V_{n} \cos \alpha_{n} \sin \phi_{n}}{r_{n}}\right) .
\end{aligned}
$$

The Cramér-Rao bound (CRB) on the variance of unbiased estimates of the sensor location are given by the diagonal elements of $\mathbf{J}^{-1}$.

We have analyzed the performance of acoustic Dopplershift estimation in [5], where we used a physics-based statistical model for the random scattering induced by turbulence in the atmosphere. CRBs are presented in [5] that illustrate the performance limits on Doppler-shift estimation 
as a function of the atmospheric conditions, the frequency of the source, and the range of the source. The turbulence scatters a fraction $\Omega \in[0,1]$ of the tone energy emitted by the source into a narrowband, zero-mean random process. The "saturation" parameter, $\Omega$, varies with the atmospheric conditions (sunny/cloudy), source frequency $\left(f_{s}\right.$ in $\mathrm{Hz}$ ), and the source range ( $r$ in meters) as

$$
\Omega=1-\exp \left(-\kappa f_{s}^{2} r\right),
$$

where $\kappa \approx 8.1 \times 10^{-7}$ for mostly sunny conditions and $\kappa \approx$ $2.8 \times 10^{-7}$ for mostly cloudy conditions. Note from (11) that the scattering becomes more severe at higher source frequencies and larger ranges. Figure 2 shows the CRB on Doppler-shift (using the model in [5]) for a scenario that is characteristic of a helicopter serving as a mobile AP: processing bandwidth $B=80 \mathrm{~Hz}$, bandwidth of scattered signal $B_{v}=1 \mathrm{~Hz}$, observation time $T=1.5 \mathrm{sec}$, and high signal-to-noise ratio (SNR). The CRBs in Figure 2 represent lower bounds on the standard deviation of Dopplershift estimates. They indicate that accuracies significantly better than $0.1 \mathrm{~Hz}$ should not be expected at these frequencies and ranges. The CRBs are not improved by higher SNR (this is the high-SNR limit, which is valid when the SNR $>20 \mathrm{~dB}$ ). We have shown in [5] that ignoring turbulent scattering leads to overly optimistic CRBs, so modeling the scattering is essential in order to get realistic performance bounds.

The FIM elements in (8)-(10) show that higher source frequency, $f_{s}$, reduces the CRB on sensor location. However, Figure 2 shows that Doppler-shift estimation is less accurate at higher source frequencies due to turbulence. Thus the additive noise variance in the model (6) may be varied with frequency (as well as range and weather conditions) to approximate the effects of these parameters on sensor location performance. In the examples that follow, we fix the standard deviation of the additive noise in (6) at $\sigma_{n}=0.1 \mathrm{~Hz}$.

\subsection{Examples}

Next we present several examples of sensor node location performance for different source paths and tone frequencies. We present CRB ellipses (defined by $\mathbf{x}^{T} \mathbf{J} \mathbf{x}=1$ ) and simulation results in which the sensor location is estimated using the nonlinear least-squares objective in (7). The source range is approximately $300 \mathrm{~m}$ in the examples, the sensor is located at the origin $\mathbf{x}_{o}=(0,0)$, the speed of sound is $c=335 \mathrm{~m} / \mathrm{s}$, and the source speed is $V_{n}=0.2 c$ (for all $n$ ). We assume that $N$ Doppler-shift measurements are available at the sensor according to the model (6). Each $\Delta f_{n}$ is assumed to be estimated at the sensor by processing a segment of acoustic time-series data with length $T^{1}$. A spectral

\footnotetext{
${ }^{1}$ The CRBs in Figure 2 are based on $T=1.5 \mathrm{sec}$.
}

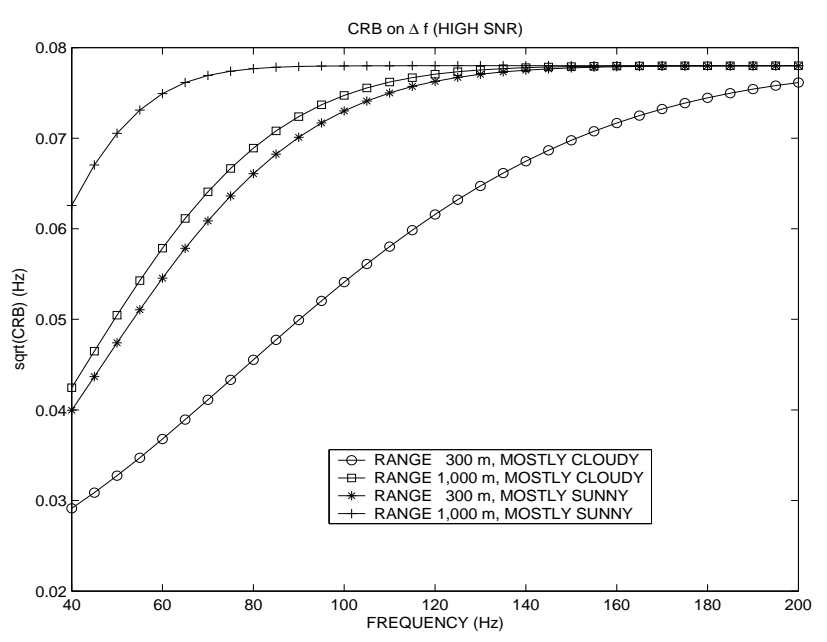

Fig. 2. Variation of CRB on Doppler-shift with weather conditions, source frequency, and source range based on the model in [5].

line tracker, e.g., Kalman filter, may be used in practice for improved accuracy compared with block processing. Also, multiple tones may be emitted by the source and tracked at the sensor, but we consider a single tone for simplicity in these examples. We set the noise standard deviation at $\sigma_{n}=0.1 \mathrm{~Hz}$ (for all $n$ ) based on Figure 2 .

In our first example, the source travels along a circular path around the sensor with radius $300 \mathrm{~m}$. The source emits a tone with $f_{s}=100 \mathrm{~Hz}$ at $N=3$ locations, with azimuth $\phi_{1}=-90^{\circ}, \phi_{2}=0^{\circ}$, and $\phi_{3}=90^{\circ}$. The source trajectory is tangential to the sensor, so $\alpha_{n}=90^{\circ}$ for $n=1,2,3$. Figure 3 shows the CRB ellipse and a scatter plot of the source location estimates for 200 runs. The source location accuracy is on the order of several meters for this scenario (the CRB ellipse radius is $1.84 \mathrm{~m}$ for one standard deviation). The standard deviation of the estimates is very close to the radius of the CRB ellipse, so the estimator very nearly achieves the CRB.

In the second example, the source travels along a straight line path parallel to the y-axis, with closest point of approach (CPA) to the sensor occurring at $x=300 \mathrm{~m}$. Again the source emits a tone with $f_{s}=100 \mathrm{~Hz}$ at $N=3 \mathrm{lo}-$ cations. The source locations are $\phi_{1}=-45^{\circ}$ (and $r_{1}=$ $424 \mathrm{~m}, \alpha_{1}=45^{\circ}$ ), $\phi_{2}=0^{\circ}$ (and $r_{2}=300 \mathrm{~m}, \alpha_{2}=90^{\circ}$ ), $\phi_{3}=45^{\circ}$ (and $r_{3}=424 \mathrm{~m}, \alpha_{3}=135^{\circ}$ ). Figure 4 shows the CRB ellipse and a scatter plot of the source location estimates for 200 runs. The accuracy is worse in this case compared with Figure 3. The CRB ellipse radius is almost twice as large in Figure 4 than in Figure 3. Note that the sensor location estimates in Figure 4 are less accurate along the $\mathrm{x}$-axis than the $\mathrm{y}$-axis, and the estimated sensor locations lie in a "cone" of uncertainty that matches the source trajectory. 


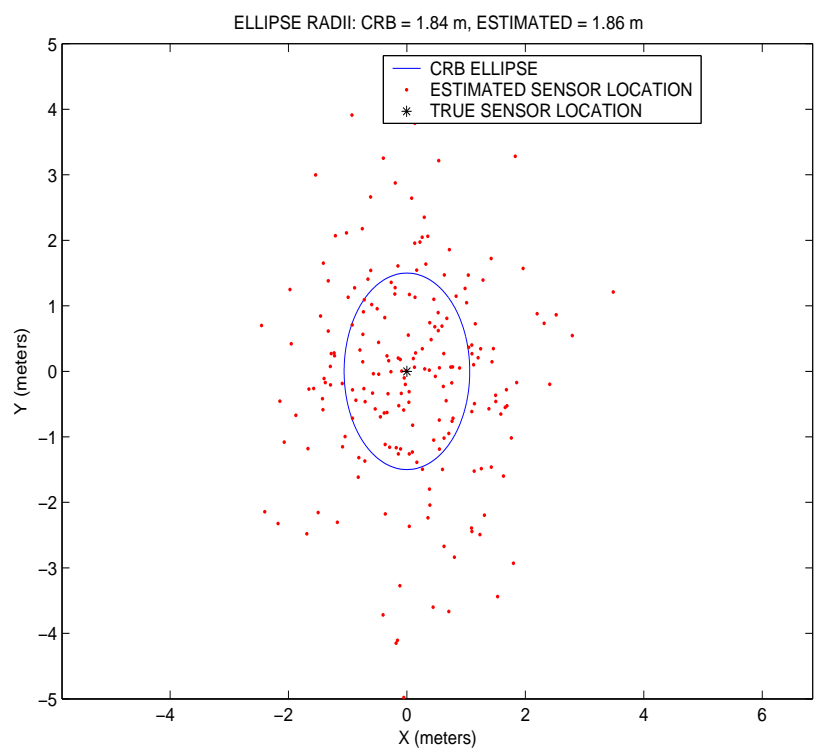

Fig. 3. CRB ellipse for sensor location and scatter plot of estimated sensor location for 200 runs: circular source trajectory around sensor.

\section{COMBINING TDE AND DOPPLER}

In this section we briefly consider the combined use of TDE and Doppler for node localization. One concurrent measurement of each is sufficient to perform localization. This is apparent from Figure 1. The TDE provides range, while Doppler provides bearing, yielding a unique solution for node location $\left(x_{0}, y_{0}\right)$. Let $s(t)$ denote the acoustic emission from the mobile AP, which we will now regard as wideband with respect to Doppler shift. Then, the noise-free received signal is

$$
s_{r}(t)=a s\left(\frac{t-\tau_{0}}{\gamma_{0}}\right),
$$

where $\tau_{0}$ is the propagation delay and $\gamma_{0}$ is the Doppler stretch factor.

Assuming an additive Gaussian noise channel with variance $N_{0}$, the CRB on estimation of $\gamma_{0}$ is [6]

$$
\operatorname{var}\left(\hat{\gamma}_{0}\right) \geq \frac{\gamma_{0} N_{0}}{2|a|^{2}} \beta
$$

where $\beta$ depends on choice of $s(t)$. (The CRB for $\tau_{0}$ decouples, and both bounds do not depend on $\tau_{0}$.) The bound on $\gamma_{0}$ becomes lower as the time-bandwidth product of $s(t)$ increases.

As an example, we computed (13) for two cases. First, with $s(t)=\cos \left(\omega_{0} t\right)$, and $\omega_{0}=200 \mathrm{~Hz}$. Second, with $s(t)$ a linear chirp spanning $[0,200] \mathrm{Hz}$. The signal energies were equal, and the time interval was one second. We

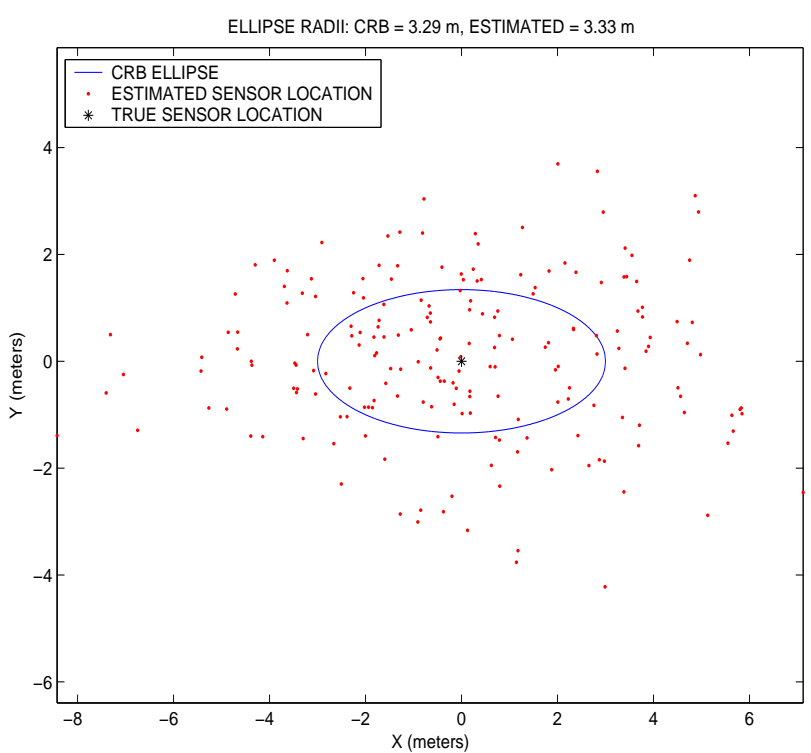

Fig. 4. CRB ellipse for sensor location and scatter plot of estimated sensor location for 200 runs: straight-line source trajectory to the right of the sensor.

find that the bound is lowered by a factor of approximately 7 for the chirp, or about $8 \mathrm{~dB}$, over that for the sinusoid. Thus, wideband signals can provide further improvement in Doppler estimation, as well as facilitating simultaneous TDE estimation. This and other combinations of processing are topics for further study.

\section{REFERENCES}

[1] R. L. Moses, D. Krishnamurthy, R. Patterson, "A selflocalization method for wireless sensor networks," Eurasip Jrnl. Appl. Sig. Proc., vol. 4, pp. 348-358, 2003.

[2] T. R. Damarla, V. Mirelli, "Sensor localization using helicopter acoustic and GPS data," Proc. of SPIE, vol. 5417, pp. 336-340, April 2004.

[3] Y. T. Chan, J. J. Towers, "Passive localization from Dopplershifted frequency measurements," IEEE Trans. Sig. Proc., vol. 40, no. 10, pp. 2594-2598, Oct. 1992.

[4] O. Cramer, "The variation of the specific heat ratio and the speed of sound in air with temperature, pressure, humidity, and $\mathrm{CO}_{2}$ concentration," Jrnl. Acoustic Soc. of Amer., vol. 93, no. 5, pp. 2510-2516, May 1993.

[5] R. J. Kozick, B. M. Sadler, "Performance of Doppler estimation for acoustic sources with atmospheric scattering," Proc. ICASSP'04, pp. 381-384, May 2004.

[6] Q. Jin, K. M. Wong, Z.-Q. Luo, "The estimation of time delay and Doppler stretch of wideband signals," IEEE Trans. Sig. Proc., vol. 43, no. 4, pp. 904-916, April 1995. 